\title{
Should we worry about bedaquiline exposure in the treatment of multidrug- resistant and extensively drug-resistant tuberculosis?
}

To the Editor:

Recently, the World Health Organization (WHO) released the updated guideline on the treatment for multi-drug resistant tuberculosis (MDR-TB) treatment regimens based on new experimental and observational evidence [1]. In this new guidance the most important drugs are the late-generation fluoroquinolones (i.e. levofloxacin and moxifloxacin), linezolid and bedaquiline. Despite the new guidance, MDR- and extensively drug resistant (XDR) TB treatment is challenging due to the risk of drug-related adverse events (AE) and drug-drug interactions (DDI). Precision medicine-based approach to minimise the risk of resistance emergence and amplification and to provide patients with the highest standard of care has been recommended [2]. Variable exposure to second-line anti-TB drugs, which has been proved in several studies, can cause either adverse events or lack of response because of too high or low concentrations, respectively. Therapeutic drug monitoring (TDM) has, therefore, been recommended to provide information on the individual drug concentration $[2,3]$. In the past decade, TDM has been frequently prescribed in well-resourced settings with promising results; on this basis, the American Thoracic Society TB guidelines have endorsed TDM in specific situations $[4,5]$. The strongest evidence on TDM in the management of M/XDR-TB is related to fluoroquinolones and linezolid [6, 7]. In vitro studies showed a clear relationship between drug exposure, susceptibility of Mycobacterium tuberculosis, and reduction in bacterial load, and cure. Observational research proved that slow therapeutic response and acquired drug resistance are likely associated with poor drug exposure. In the recently updated WHO guideline on MDR-TB treatment, TDM has been recommended for fluoroquinolones, linezolid and aminoglycosides, especially when "the dose is at the upper and lower ends of the range to minimise the adverse therapeutic consequences of over- and under-exposure" [1]. Unfortunately, randomised controlled studies on TDM have not been planned until now.

Bedaquiline-containing regimens showed their efficacy and effectiveness in experimental and observational studies. However, factors interfering with its pharmacokinetics may affect its concentrations. In particular, bedaquiline absorption is influenced by food and DDI, being metabolised by CYP450 enzymes. A post hoc analysis of the pharmacokinetic data collected in trials setup for regulatory approval showed that higher bedaquiline exposure had a beneficial effect on time to sputum culture conversion. In a subsequent covariate analysis, weight, albumin, age and race were associated with drug exposure [8]. However, strict selection criteria of the regulatory studies might have biased those results. It is key to prove the same and/ or other factors in the real-life world and their association with clinical and treatment outcomes, including the occurrence of adverse events. If such a relationship were present, this could prove the necessity of exploring the added value of TDM for bedaquiline.

The aim of the present study was to evaluate whether factors associated with altered pharmacokinetics of bedaquiline are present in a large multi-centre cohort of M/XDR-TB patients treated under different real-life conditions.

Factors known to have an impact on bedaquiline exposure are present in patients with M/XDR-TB. As we have seen with fluoroquinolones the impact of variable drug exposure needs to be studied to understand the impact on treatment outcome. http://bit.ly/2JMlJJF

Cite this article as: Alffenaar J-WC, Akkerman OW, Tiberi S, et al. Should we worry about bedaquiline exposure in the treatment of multidrug-resistant and extensively drug-resistant tuberculosis? Eur Respir $J$ 2020; 55: 1901908 [https://doi.org/10.1183/13993003.01908-2019]. 
We performed an observational retrospective study. For patient recruitment, ethical clearance, TB case definition, treatment design and study period, we refer to the original study [9]. Bedaquiline was prescribed at the licensed dose of $400 \mathrm{mg}$ once a day for 14 days, followed by $200 \mathrm{mg}$ three times a week for 22 weeks. A standardised e-form was developed and used to collect epidemiological, clinical and microbiological data from medical records. Several factors related to lower bedaquiline exposure were included in the case-report form: increased bodyweight; co-prescription of a strong inductor of CYP3A4; prior use of rifampicin for $>10$ days and stopped $<2$ weeks before the administration of bedaquiline; and uncontrolled HIV infection, diabetes mellitus and gastrointestinal complaints. Moreover, factors related to higher bedaquiline exposure were recorded: increasing age; and strong inhibitor of CYP3A4.

A total of 428 patients was available for the analysis. For a detailed description of demographics, epidemiological and clinical characteristics (including adverse events) we refer to the original study [9]. Table 1 summarises the frequency of risk factors associated with either low or high exposure.

Although administration with food is recommended, it was observed that $125(30.3 \%)$ patients received bedaquiline in a fasted condition. Administration without food reduces drug exposure of $50 \%$ [10] and may, therefore, have resulted in suboptimal exposure. In addition, bedaquiline co-administered with strong CYP3A4 inducers occurred in $81(27.2 \%)$ patients. Data on the impact of the different drug-drug interaction is scarce but it was shown that rifampicin and rifapentine reduced bedaquiline drug exposure with approximately $\sim 40-80 \%$ [11]. Moreover, simulation studies showed that efavirenz reduced drug exposure of 50\% [12]. Based on the results of these three strong inducers of CYP3A4 it can be assumed that any other strong inducer would exert a similar effect on bedaquiline exposure. Other factors that may have impacted bedaquiline exposure were also observed. Higher body weight, low albumin concentration, uncontrolled HIV infection and gastro-intestinal problems may have a detrimental effect on drug exposure but are more difficult to quantify. Even more challenging is to estimate the consequences of a combination of factors on drug exposure. Six cases, who received the drug without food but with a strong CYP3A4 inducer, showed the following treatment outcomes: four were still on treatment, one was cured and one was lost to follow-up. Our study was not the only study showing risk factors that could influence bedaquiline exposure. In the study by NDJEKA et al. [13], 200 patients received a bedaquiline-containing treatment regimen for M/XDR-TB. The cohort included 134 patients living with HIV. As part of their antiretroviral regimen, 101 patients received nevirapine, a strong CYP3A4 inductor and 34 patients received ritonavir, a strong inhibitor of $3 \mathrm{~A} 4$, resulting in either reduced or increased bedaquiline exposure. A similar situation was observed in a cohort receiving bedaquiline in combination with delamanid as compassionate use [14]. In that study, 46 of the 84 patients were co-infected with HIV potentially receiving a strong CYP3A4 inductor or inhibitor.

Factors influencing bedaquiline drug exposure are frequently present and are proved by not only our large retrospective study but also by other studies $[13,14]$. Although the impact is difficult to estimate because actual drug exposure measurements are lacking, prospective evaluation is needed to estimate the impact of

\begin{tabular}{|c|c|c|c|}
\hline Factor & Median (IQR) & Subjects n $(\%)$ & Total subjects with data $n$ \\
\hline \multicolumn{4}{|l|}{ Low } \\
\hline Body weight >80 kg & 84 (82.3-92) kg & 19 (5.3) & 359 \\
\hline Albumin $<32 \mathrm{gr} \cdot \mathrm{L}^{-1}$ & $28(25-30) \mathrm{gr} \cdot \mathrm{L}^{-1}$ & $76(22.4)$ & 340 \\
\hline Without food & & $125(30.3)$ & 413 \\
\hline Strong inducer of CYP3A4 & & $81(27.2)$ & 298 \\
\hline Prior use of rifampicin & & $9(2.2)$ & 403 \\
\hline $\begin{array}{l}\text { Uncontrolled HIV ICD4 } \\
\text { lymphocytes }\end{array}$ & 99 (44.5-159.5) & 28 (31.5) & 89 \\
\hline$<200$ cells $\cdot \mathrm{mmmc}^{-1}$ ) & & & \\
\hline Diabetes mellitus type 2 & & $26(6.3)$ & 413 \\
\hline \multicolumn{4}{|l|}{ Gastrointestinal complaints } \\
\hline Vomiting & & $87(21.2)$ & 411 \\
\hline Diarrhoea & & $56(13.6)$ & 412 \\
\hline Both & & $26(6.3)$ & 410 \\
\hline \multicolumn{4}{|l|}{ High } \\
\hline Bodyweight <50 kg & $43(38.7-46) \mathrm{kg}$ & $114(31.8)$ & 359 \\
\hline Age $>70$ years & $78(72-80)$ years & $5(1.2)$ & 428 \\
\hline Strong inhibitor of CYP3A4 & & $26(8.7)$ & 298 \\
\hline
\end{tabular}


drug-influencing factors in a real-life setting. The nature of the design should be observational and the study should be performed in an operational setting, as strong evidence from clinical trials to support therapeutic drug monitoring is lacking. Although it can be speculative, it seems plausible that at least a subset of patients might benefit from the assessment of drug exposure.

Jan-Willem C. Alffenaar ${ }^{1,2,3}$, Onno W. Akkerman ${ }^{4,5}$, Simon Tiberi $^{6}$, Giovanni Sotgiu $\odot^{7}$, Giovanni Battista Migliori $\oplus^{8,9}$ on behalf of the Global Tuberculosis Network Bedaquiline study group

${ }^{1}$ University of Sydney, Faculty of Medicine and Health, School of Pharmacy, Sydney, Australia. ${ }^{2}$ Westmead Hospital, Sydney, Australia. ${ }^{3}$ Marie Bashir Institute for Infectious Diseases and Biosecurity, University of Sydney, Sydney, Australia. ${ }^{4}$ University of Groningen, University Medical Center Groningen, Tuberculosis Center Beatrixoord, Haren, The Netherlands. ${ }^{5}$ University of Groningen, University Medical Center Groningen, Dept of Pulmonary Diseases and Tuberculosis, Groningen, The Netherlands. ${ }^{6}$ Division of Infection, Royal London Hospital, Barts Health NHS Trust, London, UK. ${ }^{7}$ Clinical Epidemiology and Medical Statistics Unit, Dept of Medical, Surgical and Experimental Sciences, University of Sassari, Sassari, Italy. ${ }^{8}$ World Health Organization Collaborating Centre for Tuberculosis and Lung Diseases, Maugeri Care and Research Institute, Tradate, Italy. ${ }^{9} \mathrm{~A}$ list of members of the Global Tuberculosis Network Bedaquiline study group can be found in the acknowledgements section.

Correspondence: Jan-Willem C. Alffenaar, University of Sydney, Faculty of Medicine and Health, School of Pharmacy and Westmead Hospital, Sydney, Australia. E-mail: johannes.alffenaar@sydney.edu.au

Received: 26 Sept 2019 | Accepted: 24 Oct 2019

Acknowledgement: The Bedaquiline study group members of the Global Tuberculosis Network are: Pablo González Montaner and Domingo Juan Palmero (Argentina); Justin Denholm, Paul Douglas, Jillian S. Lau and Adrian R. Tramontana (Australia); Alena Aleksa, Janina Artsukevich, Tatsiana Sanukevich, Alena Skrahina and Varvara Solodovnikova (Belarus); Marie-Christine Payen (Belgium); Margareth Dalcolmo (Brazil); Mina Gaga, Katerina Manika and Apostolos Papavasileiou (Greece); Rohit Amale, Shashank Ganatra, Jai Mullerpattan, Tsetan Dorji Sadutshang, Sonam Topgyal and Zarir Farokh Udwadia (India); Rosella Centis, Luigi Codecasa, Lia D’Ambrosio, Gina Gualano, Giovanni Battista Migliori, Fabrizio Palmieri, Antonella Papalia, Emanuele Pontali, Laura Saderi, Giovanni Sotgiu, Antonio Spanevello, Federica Toscanini and Pietro Viggiani (Italy); Onno Akkerman and Jan-Willem Alffenaar (Netherlands); Selene Manga (Peru); Raquel Duarte and Carlos Robalo Cordeiro (Portugal); Evgeny Belilovski, Sergey E. Borisov, Alexey Filippov and Andrey Maryandyshev (Russian Federation); Keertan Dheda, Martin Enwerem, Aliasgar Esmail, Mohammed Fadul, Barbara Lazaro Mastrapa, Jorge Lazaro Teran Troya, Suzette Oelofse and Rodolfo Romero Leyet (South Africa); Jose A. Caminero, José-María García-García, Julia-Amaranta Garcia-Fuertes and Isabel Carpena Martinez (Spain); Judith Bruchfeld, Lina Davies Forsman and Jerker Jonsson (Sweden); Heinke Kunst, Simon Tiberi, Veronica White and Alimuddin Zumla (UK).

Conflict of interest: None declared.

\section{References}

1 World Health Organization. WHO consolidated guidelines on drug-resistant tuberculosis treatment. Geneva, 2019. https://apps.who.int/iris/bitstream/handle/10665/311389/9789241550529-eng.pdf?ua=1.

2 van der Burgt EP, Sturkenboom MGG, Bolhuis MS, et al. End TB with precision treatment!. Eur Respir J 2016; 47: 680-682.

3 Ghimire S, Bolhuis MS, Sturkenboom MGG, et al. Incorporating therapeutic drug monitoring into the World Health Organization hierarchy of tuberculosis diagnostics. Eur Respir J 2016; 47: 1867-1869.

$4 \quad$ Nahid P, Dorman SE, Alipanah N, et al. Idsa 2016 (Tb). Clin Infect Dis 2017; 63: 147-195.

5 Alffenaar J, Tiberi S, Verbeeck R, et al. Therapeutic drug monitoring in tuberculosis: practical application for physicians. Clin Infect Dis 2017; 64: 104-105.

6 Sotgiu G, Centis R, D'Ambrosio L, et al. Efficacy, safety and tolerability of linezolid containing regimens in treating MDR-TB and XDR-TB: Systematic review and meta-analysis. Eur Respir J 2012; 40: 1430-1442.

7 Davies Forsman L, Bruchfeld J, Alffenaar J. Therapeutic drug monitoring to prevent acquired drug resistance of fluoroquinolones in the treatment of tuberculosis. Eur Respir J 2017; 49: 1700173.

8 Svensson EM, Karlsson MO. Modelling of mycobacterial load reveals bedaquiline's exposure-response relationship in patients with drug-resistant TB. J Antimicrob Chemother 2017; 72: 3398-3405.

9 Borisov SE, Dheda K, Enwerem M, et al. Effectiveness and safety of bedaquilinecontaining regimens in the treatment of MDR- and XDR-TB: A multicentre study. Eur Respir J 2017; 49: 1700387.

10 van Heeswijk RPG, Dannemann B, Hoetelmans RMW. Bedaquiline: A review of human pharmacokinetics and drug-drug interactions. J Antimicrob Chemother 2014; 69: 2310-2318.

11 Svensson E, Murray S, Karlsson M, et al. Rifampicin and rifapentine significantly reduce concentrations of bedaquiline, a new anti-TB drug. J Antimicrob Chemother 2015; 70: 1106-1114.

12 Svensson EM, Aweeka F, Park JG, et al. Model-based estimates of the effects of efavirenz on bedaquiline pharmacokinetics and suggested dose adjustments for patients coinfected with HIV and tuberculosis. Antimicrob Agents Chemother 2013; 57: 2780-2787.

13 Ndjeka N, Schnippel K, Master I, et al. High treatment success rate for multidrug-resistant and extensively drug-resistant tuberculosis using a bedaquiline-containing treatment regimen. Eur Respir J 2018; 52: 1801528.

14 Hafkin J, Hittel N, Martin A, et al. Compassionate use of delamanid in combination with bedaquiline for the treatment of multidrug-resistant tuberculosis. Eur Respir J 2019; 53: 1801154. 